\title{
2D ECE measurements of type-I Edge Localized Modes at ASDEX Upgrade
}

\author{
J E Boom ${ }^{1}$, I G J Classen ${ }^{1}$, P C de Vries ${ }^{1}$, T Eich ${ }^{2}$, E Wolfrum ${ }^{2}$, W Suttrop ${ }^{2}$, R P Wenninger ${ }^{3}$, \\ A J H Donné ${ }^{1,4}$, B J Tobias ${ }^{5}$, C W Domier ${ }^{6}$, N C Luhmann Jr. ${ }^{6}$, H K Park ${ }^{7}$, and the ASDEX \\ Upgrade Team \\ ${ }^{I}$ FOM Institute for Plasma Physics Rijnhuizen, 3430 BE Nieuwegein, The Netherlands \\ ${ }^{2}$ Max-Planck-Institut für Plasmaphysik, 85748 Garching bei München, Germany \\ ${ }^{3}$ Universitätssternwarte, Ludwig-Maximilians-Universität, 81679 München, Germany \\ ${ }^{4}$ Faculteit Technische Natuurkunde, Technische Universiteit Eindhoven, 5600 MB \\ Eindhoven, The Netherlands \\ ${ }^{5}$ Princeton Plasma Physics Laboratory, Princeton, NJ 08540, USA \\ ${ }^{6}$ Department of Electrical and Computer Engineering, University of California at Davis, \\ Davis, CA 95616, USA \\ ${ }^{7}$ POSTECH, Pohang, Gyeongbuk, 790-784, Korea
}

E-mail: J.E.Boom@rijnhuizen.nl

\begin{abstract}
The installation of a 2D electron cyclotron emission imaging (ECEI) diagnostic on ASDEX Upgrade has provided a new means to observe the nature of Edge Localized Modes (ELMs). For a series of ELMs in a typical type-I ELMy H-mode (with $q_{95}=4.7$ ), the 2D dynamics have been characterized. Firstly, a clear distinction between so-called 'fast' and 'slow' ELMs was found to be the occurrence of an off-mid-plane fluctuation in case of the latter. This mode has its amplitude strongest off-midplane and its poloidal and toroidal mode numbers are $m \sim 110$ and $n \sim 30$. Secondly, prior to the onset of the ELM's temperature collapse, a mode is observed that covers the whole ECEI observation window. Here, the estimated poloidal and toroidal mode numbers are $m \sim 75$ and $n \sim 20$. These have been seen to increase towards the ELM crash, simultaneously with a velocity increase of the mode (in poloidal direction). Finally, filaments have been identified during the temperature collapse phase and their motion could be followed in the vertical direction. Contrary to both the off-mid-plane fluctuation and the ELM onset mode, which only have been seen rotating in the electron diamagnetic drift direction, the first few filaments have sometimes been observed to move in the opposite direction as well.
\end{abstract}

PACS numbers: 52.55.Dy, 52.55.Fa, 52.35.Py, 52.70.Gw

\section{Introduction}

The most successful scenario for fusion reactors, as currently foreseen, is tokamak operation in high confinement mode (H-mode) [1]. However, due to the steep pressure gradients at the plasma edge, in combination with high current densities, this regime is destabilized by the occurrence of instabilities known as edge localized modes (ELMs) $[2,3]$. In case of the most commonly observed ELMs, socalled type-I, the heat load of particles expelled during these periodic relaxations of the edge transport barrier (ETB) could prove difficult to handle in future fusion devices such as ITER [4]. For the development of ELM-control tools (e.g. [5]) and for the extrapolation of models towards ITER, it is therefore crucial to understand the dynamics of the ELM cycle and its underlying physics.

In general, the type-I ELM cycle can be divided into three main phases $[6,7]$ : the first phase, at the ELM onset, is understood to be governed by the magnetohydrodynamic (MHD) stability limits within the peeling-ballooning model. This model describes, for type-I ELMs, that an increasing edge pressure gradient is limited by the onset of ballooning modes. When the edge current density continues to increase, due to the rise in bootstrap and Ohmic currents, the plasma can be driven across 
the peeling stability boundary as well. This results in a loss of edge confinement and causes the edge pressure gradient to drop, which then further destabilizes the peeling mode [8,9]. Testing of the peeling-ballooning model can be done by using numerical simulations, such as [10], for the prediction of stability limits and growth rates of the modes. These can then be compared to precursor modes that are seen just befor the ELM collapse, e.g. at ASDEX Upgrade (AUG) [11] and also on other tokamaks $[12,13]$. The second phase in the ELM cycle is non-linear of nature. It is characterized by the collapse of the temperature and density profiles at the edge, and the occurrence of helical, fieldaligned structures on the low field side (LFS). These so-called filaments are subsequently ejected from the edge and start to move into the scrape-off layer. This has been observed at spherical tokamaks [14, 15], as well as at conventional tokamaks such as AUG [16-19] or others [7, 12, 20]. Non-linear MHD simulations are focussed on modelling the development from the peeling-ballooning modes into filaments, and e.g. their interactions with the plasma flows [21]. When the second phase has ended, usually after a few ms, the ETB starts to build up again completing the ELM cycle. Characterization of typical type-I ELM cycles at AUG has revealed that so-called 'fast' ELMs get triggered immediately after recovery of the ETB, whereas for 'slow' ELMs there is an extended phase where the ETB remains in its final shape before the crash occurs [22]. However, the mechanism that triggers the onset of the next ELM is still unknown.

The installation of an electron cyclotron emission imaging diagnostic (ECEI) on AUG provides a new means to observe the 2D nature of ELM dynamics on a fast timescale, and can contribute to a better understanding in all phases of the ELM cycle. In this work, observations of the onset and development of type-I ELMs with this 2D diagnostic will be presented for the first time. First, the setup of the ECEI-system and the treatment of ECE measurements at the plasma edge will be described in section 2. After that, in section 3, the ECEI observations will be presented from a series of ELM crashes during a steady state phase in a type-I ELMy H-mode on AUG. Here, the focus will be on the first and second phase of the ELM cycle, describing the details of the crash onset and the second, non-linear decay phase. A discussion on the observations and a conclusion are given in section 4 .

\section{ECE-imaging at ASDEX-Upgrade}

In this section, a short explanation is given on the ECEI diagnostic and on its optical arrangement for measurements at the plasma edge. In the second paragraph, it is explained how the system is crosscalibrated in order to obtain electron temperature measurements. The influence of decreasing optical thickness at the edge is discussed in the last paragraph.

\subsection{Description of the diagnostic and experimental setup}

The ECEI diagnostic, as it is installed at AUG consists of an array of 16 detectors, each of which acts as a standard (1D) ECE radiometer [23-25]. That is, every detector measures the intensity of the emitted electron cyclotron radiation from different vertical positions, here in $2^{\text {nd }}$ harmonic X-mode $(100-140 \mathrm{GHz})$. Provided the plasma is optically thick, the measured power is proportional to the intensity emitted by a black body at temperature $T_{\mathrm{e}}$. The quasi-optical setup allows for the 16 lines of sight to be focussed at the LFS edge with a waist of $1.5 \mathrm{~cm}$ at the focal plane (i.e. a FWHM of 1.75 $\mathrm{cm})$. The radial coverage is determined by 8 local oscillator frequencies per line of sight, each frequency band corresponds to about $1.6 \mathrm{~cm}$ in the plasma. Hence, this matrix of 16x 8 channels covers an observation area of about $40 \times 13 \mathrm{~cm}^{2}$ with spot sizes of $1.5 \times 1.6 \mathrm{~cm}^{2}$. With the system set up to measure at the LFS edge, figures 1 and 2 show this area in a poloidal cross-section of AUG together with the 1D ECE system, which shares the same observation window. The sampling rate of ECEI is typically set to $200 \mathrm{kHz}$; the whole length of the discharge is covered at this rate. 
(3)

(1)

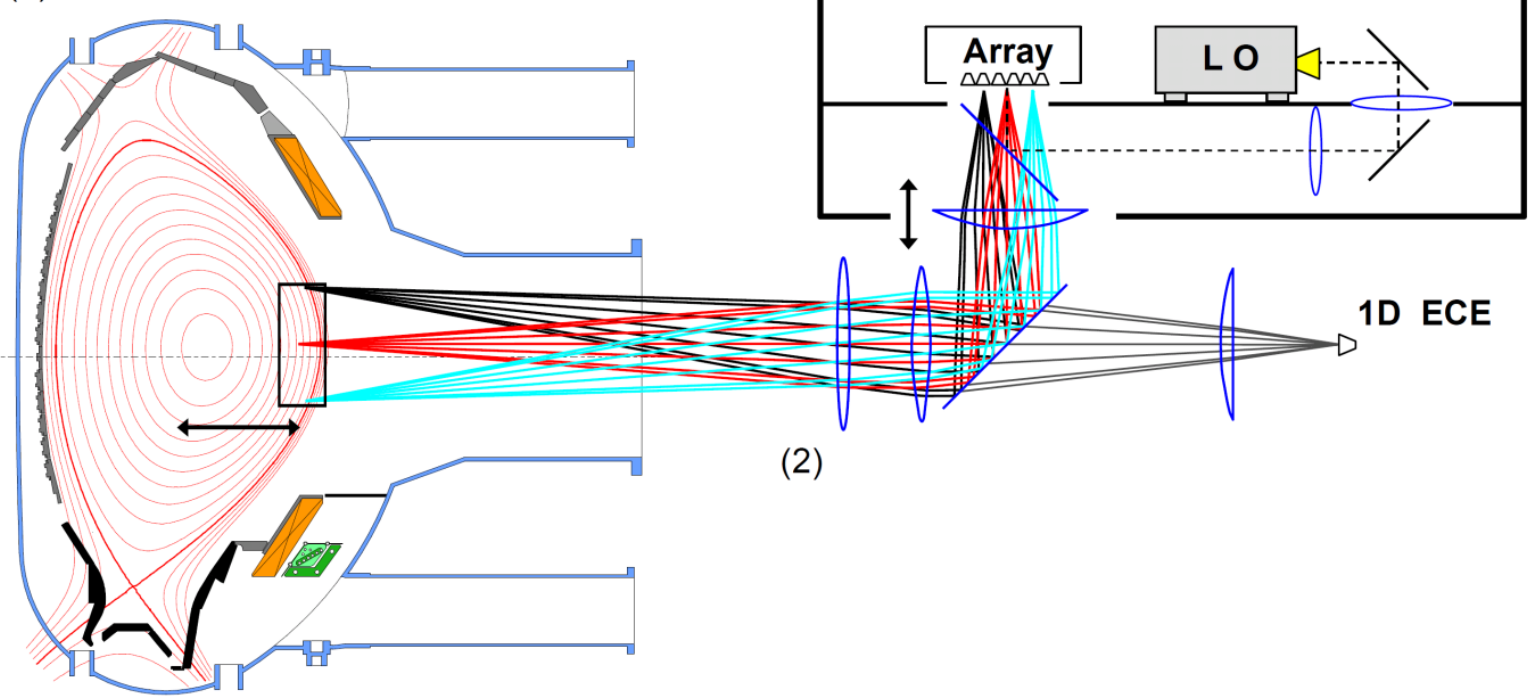

Figure 1. Schematic view of the ECEI diagnostic at AUG: a cross section of the tokamak with a standard magnetic flux surface configuration is depicted in (1). The optics of ECEI (2) consist, amongst others, of a beam splitter that separates the lines of sight of ECEI and the 1D ECE system, and a moveable lens which can be used to shift the position of the focal plane in the plasma. In (3), plasma radiation is measured by the array of 16 detectors, after being down-converted by mixing with waves from a local oscillator (LO) that are coupled in through a second beam splitter.

\subsection{Cross-calibration method}

ECEI is not independently calibrated but cross-calibrated against the 1D-ECE diagnostic, which shares the same viewing window and is absolutely calibrated using the standard hot-cold method. When the ECEI system is set up to measure at the edge, as shown in figure 2, some of the outer channels may have their ECE resonances at radii where the plasma temperatures are very low $(<100$ $\mathrm{eV}$ ), usually around the foot of the temperature pedestal. In these cases, ECEI is cross-calibrated in (other) shots with lower magnetic fields, where all channels observe regions of high temperature at high optical thickness. 


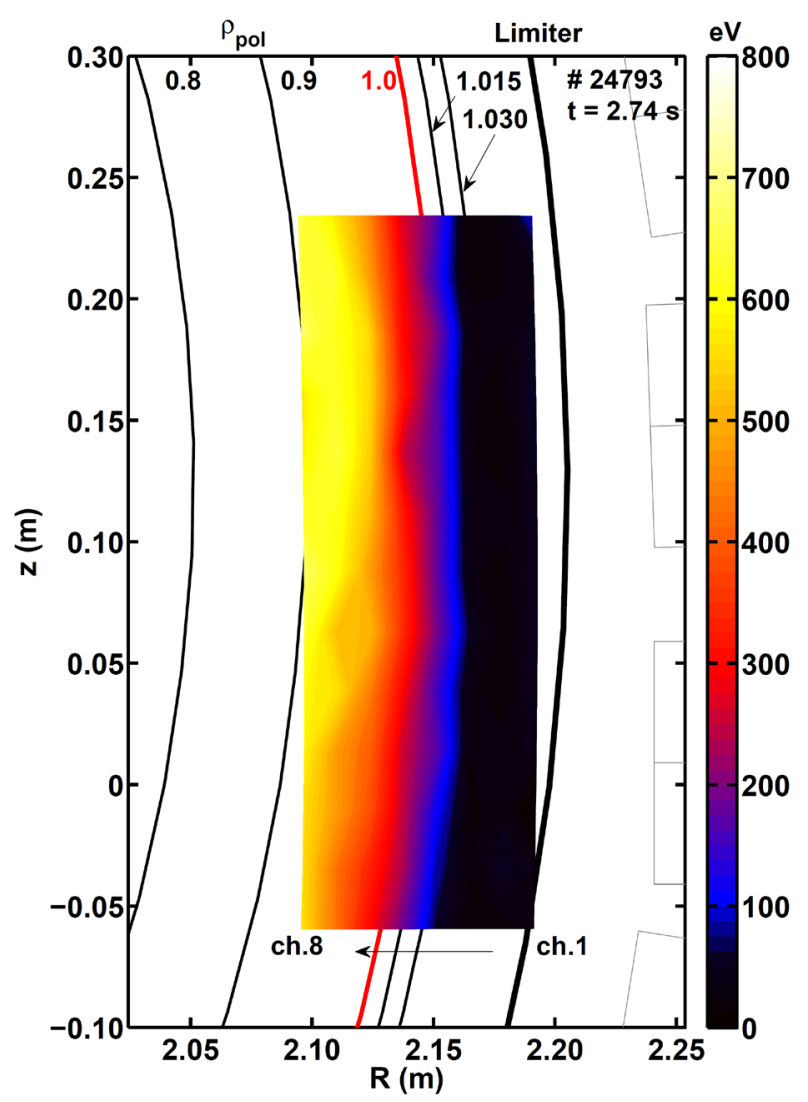

Figure 2. ECEI observation window for measurements at the LFS: the $T_{\mathrm{e}}$ profile shown is obtained using the cross-calibration method described in paragraph 2.2. It is measured during the quiet phase in between two ELMs (discharge \#24793 at $2.74 \mathrm{~s}$ ). Also shown are flux surfaces at various $\rho_{\text {pol }}$, as follow from the CLISTE equilibrium reconstruction calculated at the time point nearest to the measurement. Here, the separatrix is labelled as 1.0; at the pedestal top, $\rho_{\mathrm{pol}}=0.94, T_{\mathrm{e}}=600 \mathrm{eV}$. As indicated at the bottom, the eight channels are labelled outwards to inwards (the lines of sight are counted from top to bottom). It can also be seen that the midplane lies at about $z=0.10 \mathrm{~m}$.

\subsection{Optical thickness at the plasma edge}

In order to interpret the intensity of ECE radiation measurements in terms of local $T_{\mathrm{e}}$, and also to ensure that the radial resolution is not broadened too much, the optical thickness $\tau$ of the plasma should be sufficiently high (i.e. $\tau>3$ ). Based on the $T_{\mathrm{e}}$ and $n_{\mathrm{e}}$ profiles shown in figure 3(a) and (b), the optical thickness of the second harmonic X-mode, $\tau_{\mathrm{X} 2}$, is calculated as shown in figure 3(c) [26]. Integrated data analysis (IDA) has been used here to get the kinetic profiles both inside and outside the separatrix; where IDA combines measurements from the lithium beam and the laser interferometer for $n_{\mathrm{e}}$, and ECE for $T_{\mathrm{e}}$ [27]. The separatrix is defined where the normalised minor radius $\rho_{\mathrm{pol}}$, given by the square root of the normalised poloidal flux, is equal to unity. This calculation of $\tau_{\mathrm{X} 2}$ shown in figure $3(c)$ is a zero-dimensional approximation assuming a homogeneous plasma, with the given $T_{\mathrm{e}}$ and $n_{\mathrm{e}}$, for each radial point. The results strongly suggest that the plasma is indeed optically thick, i.e. $\tau_{\mathrm{X} 2}>3$, inside the separatrix and that radiation transport calculations are not needed to obtain a local $T_{\mathrm{e}}$ measurement. When $\tau_{\mathrm{X} 2}<3$, the optical depth is marginal and measurements of $T_{\mathrm{e}}$ could also be influenced by $n_{\mathrm{e}}$. In this case, the measurements are referred to as $T_{\mathrm{rad}}$, instead of $T_{\mathrm{e}}$. 

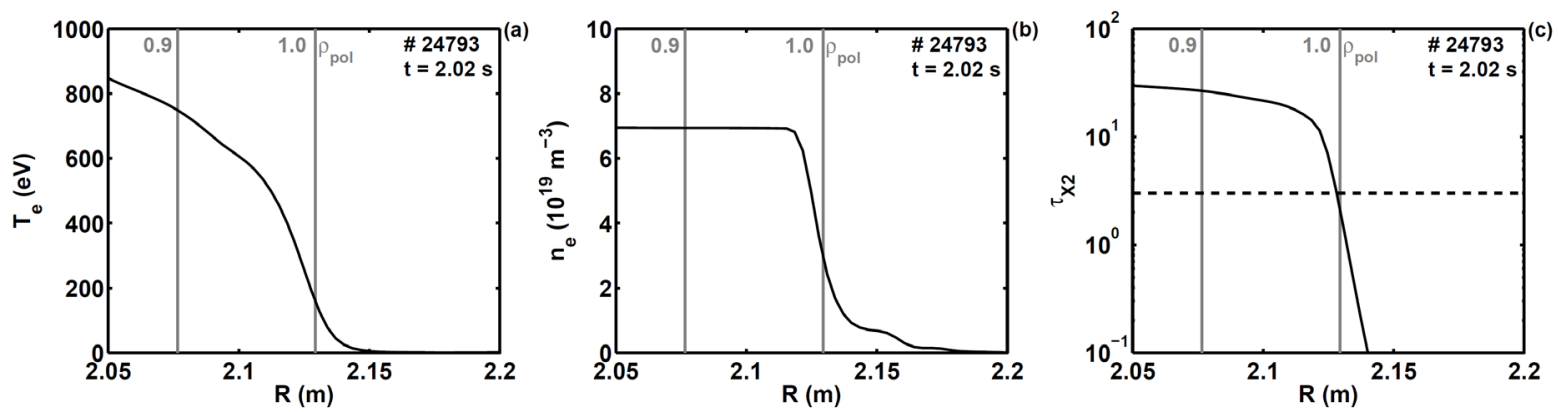

Figure 3. Using integrated data analysis for the combined measurements of the lithium beam, DCN interferometer, and ECE, $T_{\mathrm{e}}$ and $n_{\mathrm{e}}$ profiles are obtained for the quiet phase prior to an ELM (discharge \# 24793 at 2.02s), as shown in (a) and (b). Based on these profiles, the optical thickness $\tau$ of the second harmonic Xmode is calculated, as is shown in $(c)$. From this zero dimensional approximation, it can already be seen that the plasma is indeed optically thick, $\tau_{\mathrm{X} 2}>3$, inside the separatrix $\left(\rho_{\mathrm{pol}}=1.0\right)$.

\section{Characterization of the 2D electron temperature behaviour during the ELM cycle}

This section describes the ECEI observations of the 2D $T_{\mathrm{e}}$ dynamics for a series of 58 ELM crashes in one AUG discharge. The type-I ELMy H-mode, during which the measurements were taken, is introduced in the first paragraph. An overview of all the phases that are typically observed with ECEI during the ELM cycle is presented in paragraph 3.2. More detailed descriptions of the three main phases identified, are given in the following three paragraphs.

\subsection{Discharge description}

With the ECEI observational area positioned at the LFS plasma edge, as shown in figure 2, the $2 \mathrm{D} T_{\mathrm{e}}$ evolution of the ELM cycle can be observed in detail. For this purpose, a typical type-I ELMy Hmode AUG discharge was used (\#24793), which has a phase of about $0.8 \mathrm{~s}$ where the main plasma parameters were kept constant. The discharge had a plasma current of $I_{\mathrm{p}}=1.0 \mathrm{MA}$, a magnetic field of $B_{\mathrm{t}}=-2.5 \mathrm{~T}$, and an edge safety factor of $q_{95}=4.7$. The edge $T_{\mathrm{e}}$ and $T_{\mathrm{i}}$ were 500 and $700 \mathrm{eV}$, both measured at $R=2.10 \mathrm{~m}$. The core line-integrated electron density was $n_{\mathrm{e}}=8 \cdot 10^{19} \mathrm{~m}^{-2}$ and the lineintegrated edge density (up to $\rho_{\mathrm{pol}} 0.8$ ) was $6 \cdot 10^{19} \mathrm{~m}^{-2}$. The plasma was heated by $7 \mathrm{MW}$ from neutral beam injection $(\mathrm{NBI})$, and $750 \mathrm{~kW}$ from electron cyclotron resonance heating (ECRH). An overview of the time traces of this discharge is given in figure 4, where also the analysed time window $(t=2.0-$ $2.8 \mathrm{~s}$ ) is indicated. Over the second half of this time window, from 2.4-2.8 s, the plasma is slightly shifted outwards by $1 \mathrm{~cm}$, figure $4(f)$, but the plasma edge remains well covered by the ECEI observation window. The degradation in energy confinement from $3.12 \mathrm{~s}$ onwards, figure $4(\mathrm{~g})$, is caused by the occurrence of a large core magnetic island. The analysed time window is chosen such that any influence of this event on the observations at the edge is prevented. 


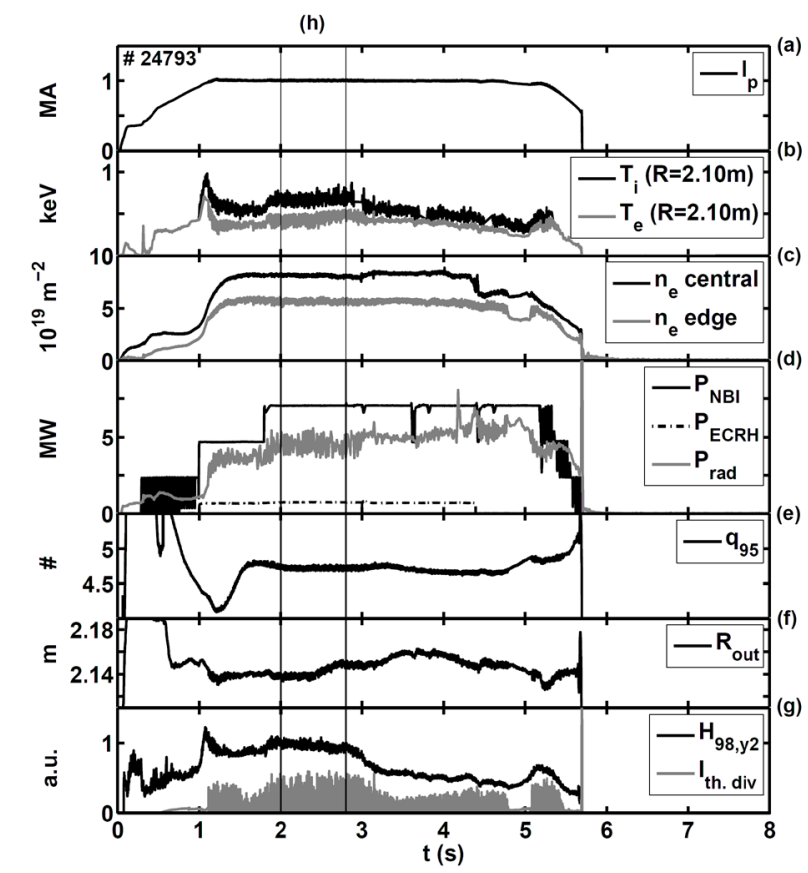

Figure 4. Overview of AUG discharge \#24793: (a) plasma current, (b) ion and electron temperatures near the edge (at major radius $R=2.10 \mathrm{~m}$ ), (c) line-averaged electron density and density at the edge, (d) input powers of neutral beam injection and electron cyclotron resonance heating, and radiated power, (e) safety factor at the edge, $(f)$ position of the outer major radius of the confined plasma, $(g)$ ratio of the thermal energy confinement time to the ITER 98(y,2) H-mode scaling value and the thermo-current in the outer divertor, $(h)$ indication of the steady state time window $(t=2.0-2.8 \mathrm{~s})$ in which the ELMs are analyzed.

The time trace of the thermo-current in the outer divertor, i.e. when the heat pulse due to the ELM reaches the divertor, is used as an indicator for occurrences of ELMs as shown in figure 5. The start time of an ELM crash is defined as the first time a predefined offset value in the thermo-current is transcended, and from this the ELM frequency is determined. As can be seen from figure 5, the majority of the crashes come with a frequency (i.e. reciprocal time) of $67 \pm 6 \mathrm{~Hz}$ but about $25 \%$ come with a frequency of more than $80 \mathrm{~Hz}$. The two different cycles are known as so-called 'slow' and 'fast' ELMs [22]. As will be discussed in detail in paragraphs 3.2 and 3.3, a specific off-mid-plane temperature fluctuation has been observed with ECEI that occurs for all (but one) of the 'slow' ELMs, which was not the case for any of the 'fast' ELMs.

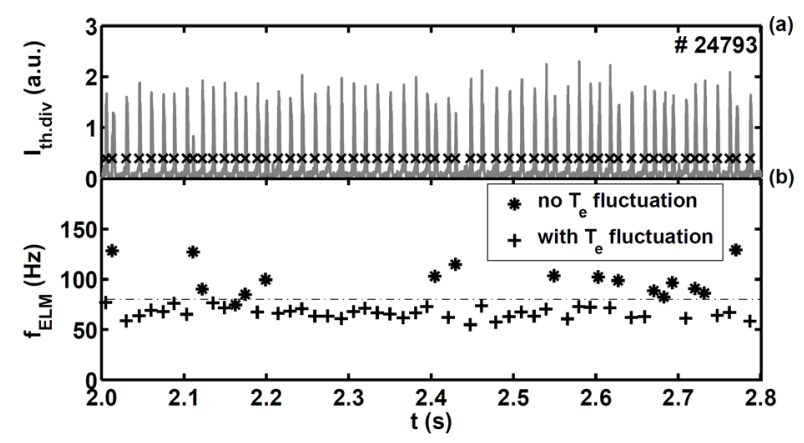

Figure 5. In (a), the time trace of the thermo-current in the outer divertor is shown for the steady state phase of AUG discharge \# 24793 (2.0-2.8 s). The ELM frequency, as determined when the thermo-currents peak above a preset level, is shown in (b). It can be seen here that the ELMs come with two distinct frequencies: for all ELMs below $80 \mathrm{~Hz}$ ('slow' ELMs), with one exception, an off-mid-plane temperature fluctuation is observed, whereas this was not the case for any of the ELMs faster than $80 \mathrm{~Hz}$ ('fast' ELMs).

\subsection{Overview of the ELM cycle}

In order to quickly obtain an impression of the spatiotemporal behaviour of phenomena that occur during an ELM cycle, the measurements from ECEI have first been averaged radially. This is done by 
dividing the area that is covered by the ECEI observation window into two regions, and taking the radial average $\left\langle T_{\mathrm{e}}\right\rangle_{\mathrm{R}}$ of the inner four and outer four channels. Of course, the radial resolution is lost by taking this average. On the other hand, the features of interest mostly occur either inside the separatrix (inner four channels, $R=2.09-2.13 \mathrm{~m}$ ) and prior to the crash, or outside (outer four channels, $R=2.15-2.19 \mathrm{~m}$ ) and during the crash. This method, therefore, does provide a simultaneous impression of the largest deviations from $<T_{\mathrm{e}}>$ as a function of the vertical, or poloidal, direction and time.

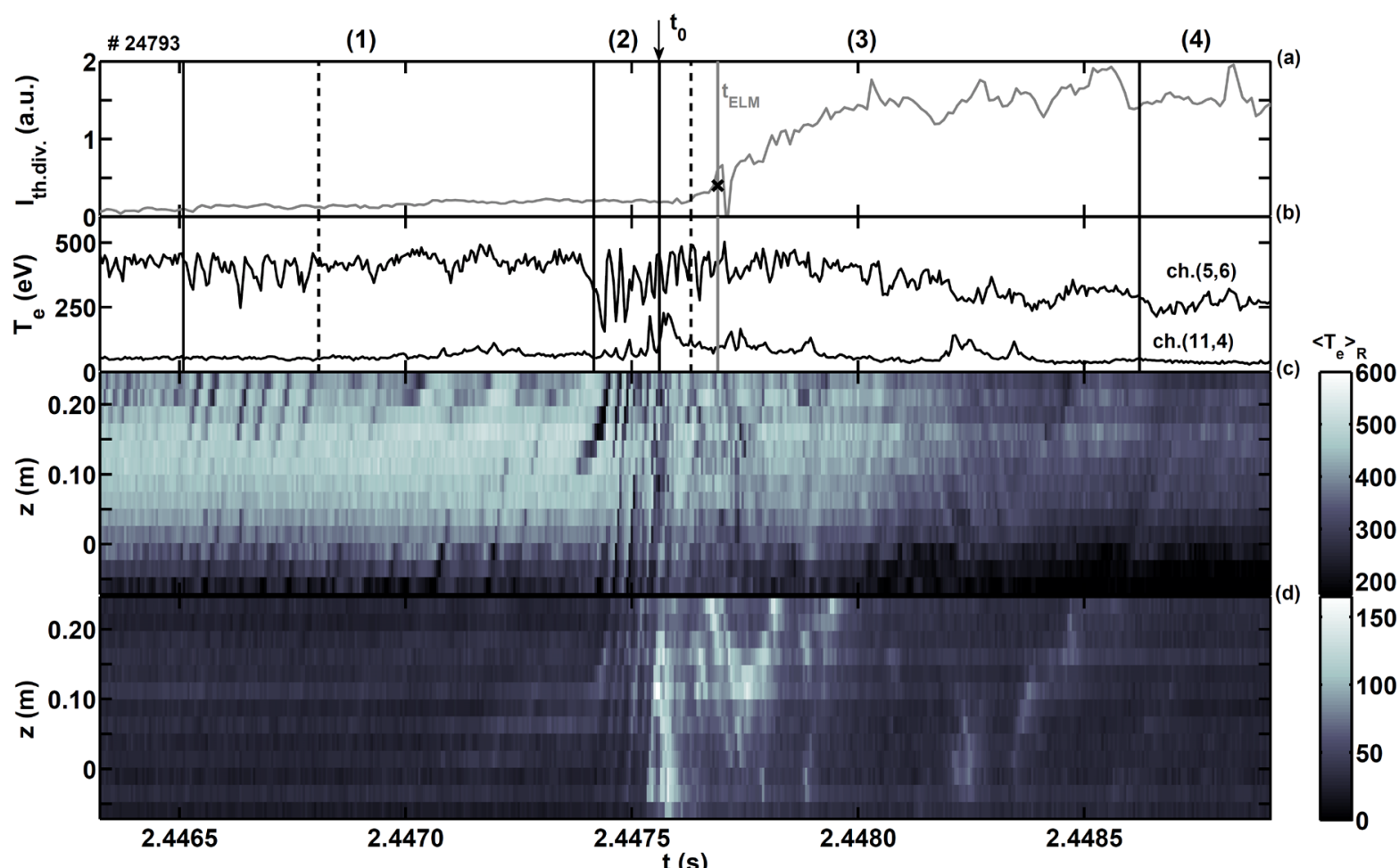

Figure 6. Overview of a typical ELM cycle: in $(a)$ the divertor thermo-current signal is shown, from which the time $t_{\mathrm{ELM}}$ is generally used as an indicator for the start of the ELM. Two $T_{\mathrm{e}}$ time-traces of different ECEI channels are shown in $(b)$ : ch. $(5,6)$ measures at $R=2.12 \mathrm{~m}, z=+0.16 \mathrm{~m}$, above the mid-plane and just inside the separatrix at the top of the pedestal. The measurement location of ch. $(11,4)$ is $R=2.15 \mathrm{~m}, z=-0.01 \mathrm{~m}$, i.e. below the mid-plane and just outside the separatrix. The $\left\langle T_{\mathrm{e}}\right\rangle_{\mathrm{R}}$ measurements are shown in (c) and $(d)$; in $(c)$ the $<T_{\mathrm{e}}>_{\mathrm{R}}$ of the inner four channels is shown, and in $(d)$ that of the outer four. From $(c)$ and $(d)$, the different phases of the ELM cycle are identified: (1) indicates the phase where the off-mid-plane fluctuation is observed. During phase (2), a mode is seen that leads up to the ELM crash at $t_{0}$. The ELM crash phase (3), during which $T_{\mathrm{e}}$ collapses, consists of a 'chaotic' stage at the beginning, followed by a series of filaments. In order to reveal the dynamics of these earlier stages, the largest part of the recovery phase (4) is omitted in this figure.

Using the two sets of radially averaged temperatures, all ELMs in the $t=2.0-2.8 \mathrm{~s}$ time-slice of the discharge shown in figure 4 have been analyzed. From this, the following short description of a typical 'slow' ELM cycle is obtained. An example of such an ELM is shown in figure 6, where features moving poloidally can clearly be seen as moving upwards in the graphs (figure 6(c) and $(d)$ ). From these graphs, the velocity with which these features move can be determined from the slope. The different phases that have been identified are indicated by the numbers in brackets on top of figure 6 .

For all observed 'slow' ELMs, the first sign of an upcoming crash is found as a temperature fluctuation which appears about $1.7 \mathrm{~ms}$ before the final crash (phase (1), figure $6(\mathrm{~b})$ and $(c)$ ), and which occurs markedly strongest off-mid-plane. Before the final ELM crash happens, a more quiescent phase sets in: during this phase the regular off-mid-plane oscillations have disappeared, but more often than not irregular temperature perturbations can be seen. It is this off-mid-plane fluctuation, and the following quiescent phase, that have not been observed for any of the 'fast' 
ELMs. The next phase (phase (2), figure 6(b) and (c)) of the ELM cycle is the phase which leads up to the crash itself. A mode is observed over the whole height of the ECEI window, usually rotating faster than the earlier off-mid-plane fluctuation. This phase usually lasts just $100 \mu \mathrm{s}$ and the end of this mode is defined here as the start $\left(t=t_{0}\right)$ of each ELM crash, which occurs before the $t_{\mathrm{ELM}}$ indicated by the thermo-currents (figure 6(a)). Directly following the second mode the actual crash starts (phase (3)). It starts off with ECEI signals which behave chaotically and are difficult to interpret, followed by a phase that shows features passing by on the outer channels of the ECEI observation window, here identified as filaments. Simultaneously, the temperature also collapses on the inner channels, figure 6(b) and (d). After no more filaments occur on the outer channels, $T_{\mathrm{e}}$ has reached its minimum on the inner channels and the ETB starts to build up again, completing the ELM cycle (phase (4)). In the next three paragraphs, the characteristics of the phases 1-3 described above will be discussed in more detail.

\subsection{Off-mid-plane fluctuations and quiescent phase}

As just described in paragraph 3.2, a distinct off-mid-plane fluctuation and quiescent phase were found to precede the crash for almost all 'slow' ELMs.

This $T_{\mathrm{e}}$ fluctuation is always seen to appear first and strongest above the mid-plane (on average 1.7 $\mathrm{ms}$ before the final crash), but if the phase lasts long enough it can be seen below the mid-plane as well (for $17 \%$ of the 'slow' ELMs). An example of this is shown in figure 7. Notably its amplitude has disappeared around the mid-plane. The duration of this phase is $0.15-1.70 \mathrm{~ms}$ and the movement of the off-mid-plane fluctuations is always directed upwards in the ECEI frame, i.e. in the electron diamagnetic drift direction, with velocities of $2-4 \mathrm{~km} / \mathrm{s}$. The fluctuation, with frequencies in the range of $20-50 \mathrm{kHz}$, manifests itself as a series of temperature dips and returns to the previous values, rather than as an oscillation around the previous temperature. This can be seen from the time-traces in figure $7(a)$. The temperature dips can be as large as $60 \%$ of the pre-oscillation value and are even seen to reach values below those observed at the end of the following ELM crash. 


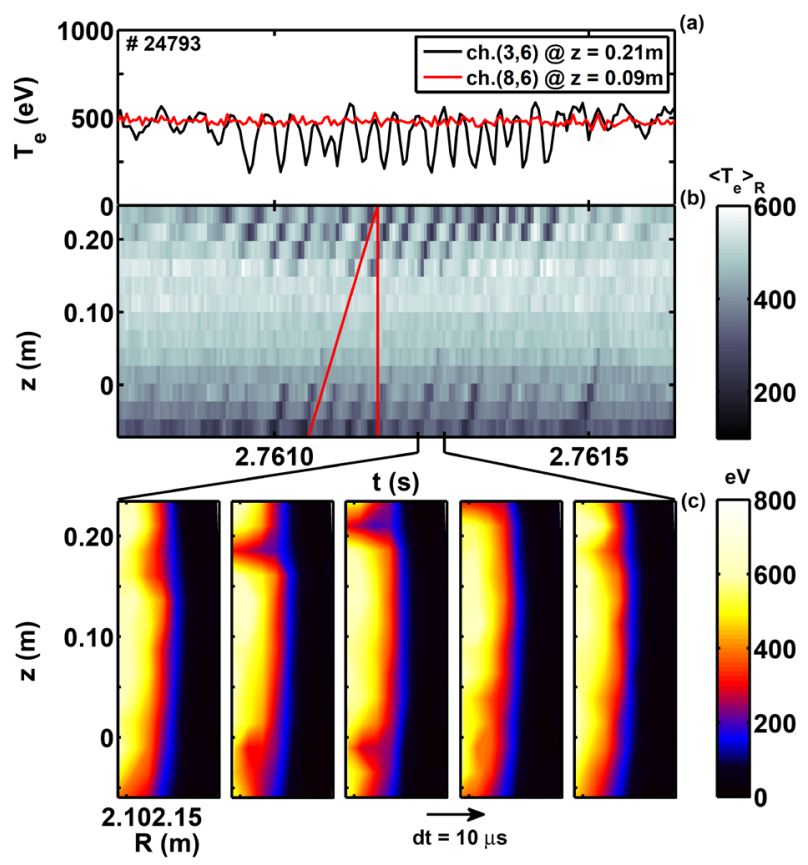

Figure 7. Example of an off-mid-plane fluctuation before an ELM crash: in (a), the time-traces of two ECEI channels are shown, where both channels measure inside the separatrix at the same major radius $(R=2.12 \mathrm{~m})$. No $T_{\mathrm{e}}$ fluctuations are observed just below the mid-plane, where ch. $(8,6)$ measures. Whereas ch. $(3,6)$, measuring about $10 \mathrm{~cm}$ above the mid-plane, clearly shows the off-mid-plane fluctuation appearing and disappearing again. Shown in (b) are the $\left\langle T_{\mathrm{e}}\right\rangle_{\mathrm{R}}$ measurements of the inner four channels; comparable to figure $6(c)$ but only showing the time-window of an off-mid-plane oscillation. In this case, it can be seen that this fluctuation appears both above and below the mid-plane. As is deduced from the two straight lines, 3 periods fit into the ECEI observation window. The five panels in (c) show the $2 \mathrm{D} T_{\mathrm{e}}(\mathrm{eV})$ evolution of this fluctuation.

The radial extension or position of this mode activity is not easily determined from the ECEI measurement and it is most likely to extend radially inwards with respect to the observation area. Therefore, as a first estimate, the poloidal mode number $m$ is estimated assuming that the mode is located on the $q=4$ surface. From the off-mid-plane fluctuation shown in figure 7(b), it is estimated that 3 periods fit into the ECEI observation window, which has a height of $0.29 \mathrm{~m}$. Next, the straight field line approximation has to be taken into account, which corrects for the fact that the derivative of the poloidal angle $\theta$ does not in general have the same direction as the derivative of the poloidal magnetic field [28]. This holds especially for the non-circular cross section of a flux surface in Xpoint configuration, which is the case here, and this method provides a poloidal angle $\theta^{*}$ which can be linearly extrapolated. As is determined from the straight field line angle calculation of $\theta^{*}$ shown in figure 8 , the ratio $\mathrm{d} \theta / \mathrm{d} \theta^{*}$ is about 3.65. From this, the poloidal mode number $m$ is estimated to be 112 \pm 12 and, accordingly, the toroidal mode number $n=28 \pm 7$. Here, the error margins originate from assuming the location of the mode to be on the neighbouring $q=7 / 2$ and $9 / 2$ flux surfaces. 


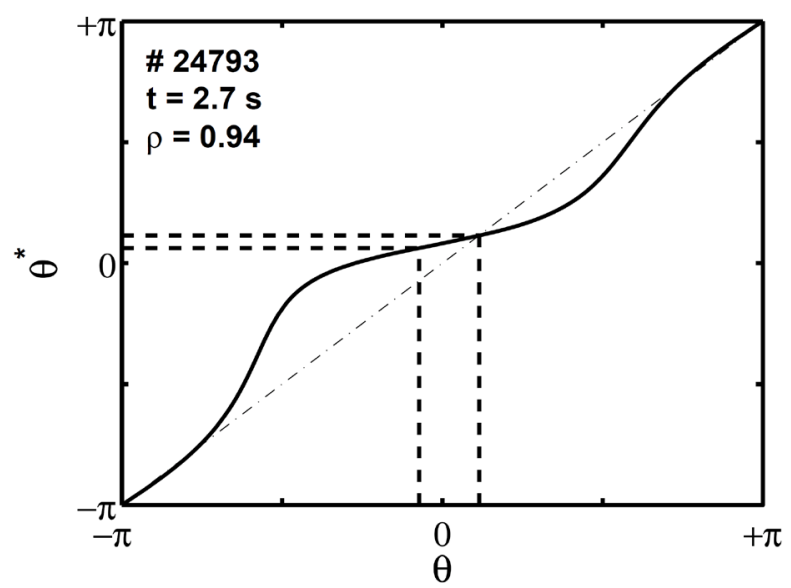

Figure 8. Relation of the straight field line angle $\theta^{*}$ to the poloidal angle $\theta$ : based on the CLISTE equilibrium reconstruction that is nearest in time to the shown measurements, the relation of $\theta$ and $\theta^{*}$ is determined for the normalized radius $\rho_{\mathrm{pol}}=0.94$ (where the centre of the mode is estimated to be, and the $q=4$ surface is located). The ratio of $\mathrm{d} \theta / \mathrm{d} \theta^{*}$ is 3.65 , as is deduced from the dashed lines.

As the off-mid-plane oscillations decrease in amplitude again, the plasma enters a quiescent phase (for $0.65 \mathrm{~ms}$ on average) where temperatures hover around the same levels as before. In many of the analyzed cases, however, the quiescent phase is disturbed by irregular occurrences of single temperature dips, sometimes reminiscent to those seen during the off-mid-plane fluctuations.

\subsection{Mode structure at the ELM onset}

As is indicated in figure 6, the quiescent phase is followed by a phase of clear deformation of the temperature profile inside the separatrix. It is only at the end of this phase that the temperature finally starts to decrease. Here, this end is defined as the start time of the ELM crash. Due to the fact that the ECEI observation window is located at the outer mid-plane, this moment occurs even before the thermo-currents in the divertor start to rise: on average $160 \mu$ s for 'slow' ELMs and $210 \mu \mathrm{s}$ for 'fast' ELMs. For most 'slow' ELMs the observed deformation shows a coherent poloidal mode structure which lasts on average $80 \mu \mathrm{s}$. For the 'fast' ELMs, their occurrence lasts only $30 \mu \mathrm{s}$ on average. A typical example of such a mode is shown in figure 9. However, since different variations of the evolution of this mode have been observed, a short discussion of the variations is given at the end of this paragraph.

Similar to the previously discussed off-mid-plane fluctuation, the direction of this mode's rotation is always upwards in the ECEI frame, i.e. in the electron diamagnetic drift direction. For the typical case shown in figure 9, the observed mode has a poloidal wavelength of ca. $15 \mathrm{~cm}$ and a radial displacement of almost $3 \mathrm{~cm}$. 


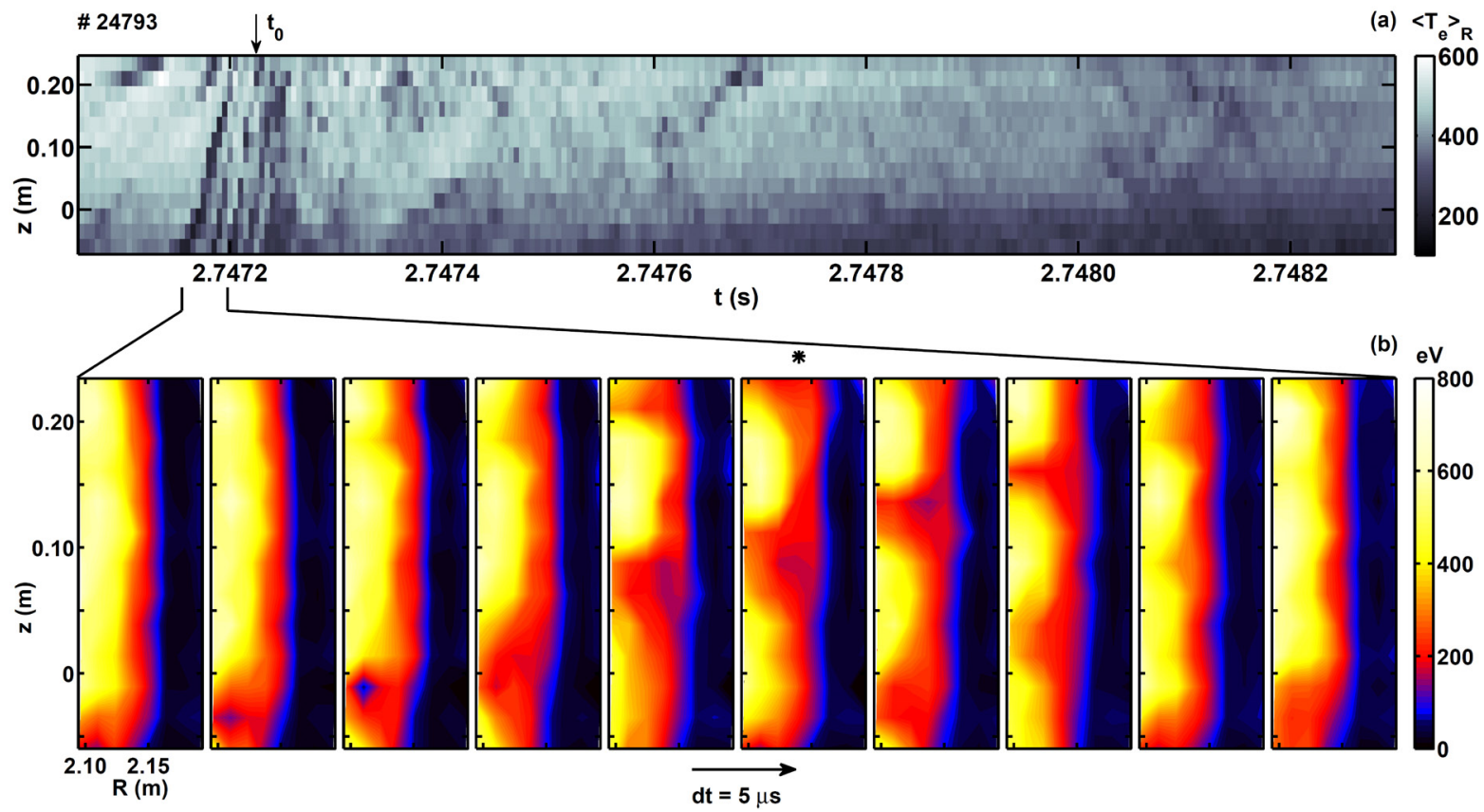

Figure 9. Mode development at the ELM onset: in (a), similar to figure $6(c)$, the $\left\langle T_{\mathrm{e}}>_{\mathrm{R}}\right.$ of the inner four channels is shown as a function of the vertical position and time. Here, it can be seen that the mode occurs about $75 \mu$ s before the ELM crash at $t_{0}$. The $<T_{\text {rad }}>_{\mathrm{R}}$ for the same time-window, but then of the outer four channels, is shown in figure 10. The ten panels in (b) are the consecutive frames (with $\mathrm{d} t=5 \mu \mathrm{s}$ ) that show the $2 \mathrm{D} T_{\mathrm{e}}(\mathrm{eV})$ evolution of the mode. The frame in $(b)$ indicated with $*$ is used for the estimate of the mode numbers.

Based on the 2D $T_{\mathrm{e}}$ measurement in figure $9\left(b^{*}\right)$ and the CLISTE equilibrium reconstruction that is nearest in time, the poloidal mode number $m$ can be calculated. Using the straight field line approximation (as described in section 3.3 and cf. figure 8), the poloidal mode number $m$ is estimated to be $74 \pm 9$ and, accordingly, the toroidal mode number $n=18 \pm 4$. Again, the error margins originate from assuming the location of the mode to be on the neighbouring $q=7 / 2$ and 9/2 flux surfaces.

In $50 \%$ of the observed 'slow' ELM crashes, including the one shown in figure 9, the mode is seen to increase in velocity towards the end of its life time. These modes start with a velocity of $4-5 \mathrm{~km} / \mathrm{s}$, which is similar to that of the preceding off-mid-plane fluctuation, and then increase to at least 7-9 $\mathrm{km} / \mathrm{s}$. Notably, from figure $9(b)$, it is also seen that the mode number clearly increases in time; increasing as much as $50 \%$ in $10 \mu$ s towards the crash.

The description of the ELM onset mode as given above applies to the majority of ELM crashes. However, some variations have been observed as well. From all analyzed 'slow' ELMs (41), six crashes show different dynamics: three 'slow' ELMs have shown a quiescent phase after a mode that looked similar to the ELM onset mode described above. In three other cases a mode was not even seen at all. Considering the 'fast' ELMs (17 in total), seven cases were found that showed different dynamics: a similar quiescent phase, occurring after an ELM onset-like mode, was observed five times, and twice there was no mode at all.

\subsection{ELM crash and filaments}

After the occurrence of the mode described in paragraph 3.4, the ELM crash phase starts: a chaotic phase comes first, where the coherent mode structures can no longer be identified (although sometimes remnants of the ELM-onset mode can be seen) and the temperatures of the inner ECEI channels eventually begin to collapse. This is followed by a phase where structures of increased temperature, so-called filaments, occur on the outer channels. It is only after the last filament has occurred, on average $1.6 \mathrm{~ms}$ after the ELM crash has started, that the temperatures in the inner channels stop decreasing and the recovery phase starts. 
For 'slow' ELMs, the chaotic phase lasts on average $150 \mu \mathrm{s}$, and for 'fast' ELMs this is a bit shorter with $95 \mu \mathrm{s}$. The end of the chaotic phase is almost always marked by the absence of any activity in the whole ECEI-observation window, or sometimes by the clear occurrence of the first filament.

For the ELMs analyzed here, all structures appearing after the chaotic phase have been identified as filaments when an increased $\left\langle T_{\mathrm{rad}}\right\rangle_{\mathrm{R}}$ (as described in paragraph 2.3) could be followed for a longer period of time and/or over multiple channels in the vertical direction. For both 'slow' and 'fast' ELMs, seven filaments on average could be identified after each ELM crash; with minimum and maximum numbers of 2 and 13. The first filament is usually seen within the first $200 \mu$ s after the end of the chaotic phase; depending on the number of filaments, the last filament can occur up to $2 \mathrm{~ms}$ after the first. The number of filaments that can be seen depends on the length of the temperature crash phase.

The measurements in figure 10 show an example of a filament; in this case one that travels through the whole of the ECEI observation window during the cause of its lifetime. These measurements are taken following the exact same ELM onset mode that was described in the previous paragraph and they show the filament as a poloidally localized structure of increased local temperature around the very edge. The filament has a height of $7 \mathrm{~cm}$ and rotates in the upward direction with a velocity of ca. $2 \mathrm{~km} / \mathrm{s}$. In order to enhance this structure, since it lies in the steep gradient region, $T_{\text {rad }}-T_{\text {rad,avg }}$ is shown in figure $10(b)$ rather than the absolute (radiation) temperature $T_{\text {rad }}$. Here, $T_{\text {rad,avg }}$ is the average radiation temperature taken at the end of the crash. Assuming locally increased electron densities [29] inside a filament, the optical thickness inside the structure could (marginally) be above unity [23]. So, although these measurements are obtained from regions just at and outside the separatrix, the radiation temperatures (with maxima up to $200 \mathrm{eV}$ ) are still assumed to be strongly correlated to the electron temperature.

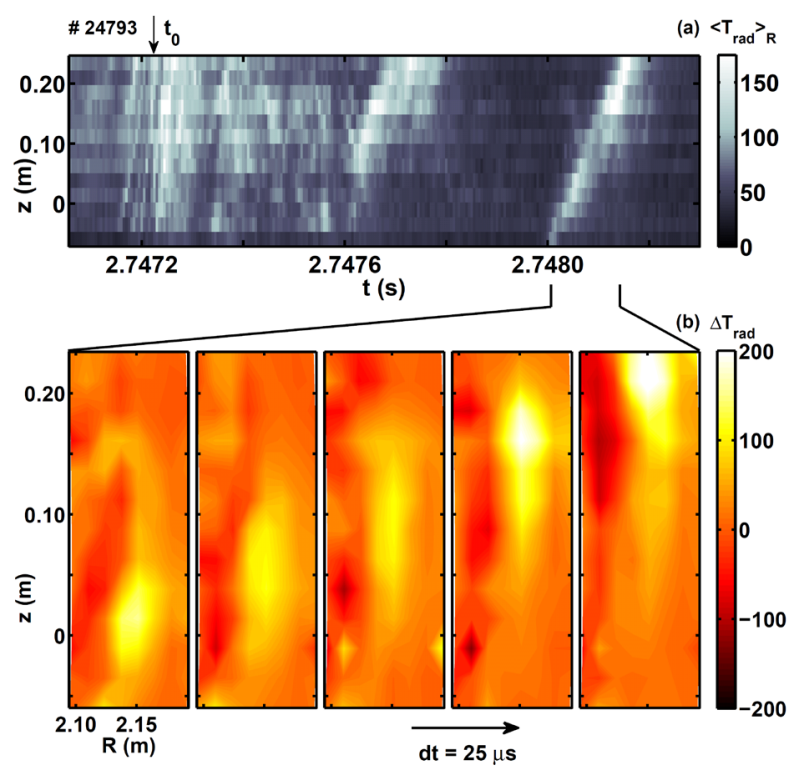

Figure 10. Example of a filament: in $(a)$, similar to figure $6(d)$, the $\left\langle T_{\text {rad }}\right\rangle_{\mathrm{R}}$ of the outer four channels is shown as a function of $z$ and time; this is, in fact, for the same time-window a shown in figure 9. Here, it can be seen that at $t=2.7480 \mathrm{~s}$ a filament starts to move from bottom to top. In (b), five panels show the 2D measurements of this filament as it passes through the ECEI observation window; here, in order to enhance the structure, $\Delta T_{\text {rad }}=T_{\text {rad }}-T_{\text {rad,avg }}$ is shown where $T_{\text {rad,avg }}$ is determined after the filament has passed.

Usually the filaments move in upward direction with a typical speed of a few $\mathrm{km} / \mathrm{s}$. However, in 20 ELM crash phases ( 15 'slow' and 5 'fast' ones), filaments have been observed to move in the opposite direction of the preceding ELM onset mode. In all these cases, it is observed that this downward movement only concerns the first filament, or first few, that occur after the 'chaotic' phase. 


\section{Discussion and conclusion}

As has been shown, the 2D aspect of ECEI (and its fast measurement) has revealed several features of ELM dynamics that were previously inaccessible to ECE measurements. Firstly, it has been seen that a difference between 'slow' and 'fast' ELMs at AUG is the occurrence of a temperature fluctuation which has its amplitude strongest off mid-plane and is followed by quiescent phase. Its poloidal and toroidal mode numbers are $m=112 \pm 12$ and $n=28 \pm 7$. Secondly, at the onset of the ELM crash, and a few tens of $\mu$ s before the ELM crash is observed by the thermo-currents in the divertor, a mode is observed covering the entire ECEI observation window. The estimated mode numbers are $m=74 \pm 9$ and $n=18 \pm 4$, which, most remarkably, are seen to increase towards the crash. Simultaneously, the velocity (in the poloidal direction) of the mode increases. Finally, during the temperature collapse phase, ELM filaments have been identified in the outer half of the ECEI observation window and their motion can be followed in the vertical direction. Contrary to both the off-mid-plane fluctuation and the ELM onset mode, which only have been seen rotating in the electron diamagnetic drift direction, sometimes the first few filaments have been observed to move in the opposite direction as well. Observations like these clearly show the benefit of the 2D ECEI diagnostic, since such features could not independently have been derived from 1D measurements.

With respect to the classification of the observed phases, it has to be stressed here that this is based on the analysis of a single, albeit typical, AUG type-I ELMy H-mode discharge with a $q_{95}$ of 4.7. In order to characterize the whole series of ELMs, the dimensionality of the ECEI measurements $\left(T_{\mathrm{e}}\right.$ as a function of $R, z$, and time) has been brought down by applying radial averaging over two halves of the observation window. The application of this averaging method, which enhances the radially coherent features, might also have suppressed incoherent features that could still be relevant.

With regard to the off-mid-plane fluctuation, which has been found here to be a distinguishing factor between 'slow' and 'fast' ELM cycles, the following has to be taken into account. It was shown in [22] that for 'slow' ELMs all edge profiles reached their final shape up to $7 \mathrm{~ms}$ before the final crash. But since the observed off-mid-plane fluctuation occurs only in the last $2 \mathrm{~ms}$, it cannot be the sole cause of the delay; also in the discharge analyzed here, the difference between 'slow' and 'fast' ELMs was on average $5 \mathrm{~ms}$. However, the presence of this fluctuation does suggest that it regulates the plasma edge condition in such a way that a stable situation is prolonged at least a little longer until the ELM crash finally comes.

Due to the quiescent phase that often occurs in between the off-mid-plane temperature oscillation and the onset mode, seen just prior to the start of the temperature crash, it is not clear whether or not this off-mid-plane fluctuation develops into the ELM onset mode, or if the two are separate phenomena. The mode numbers that have been estimated are, however, of the same order of magnitude, with those for the off-mid-plane oscillation being slightly higher. With respect to the fast changes in mode number and velocity that have been observed for the ELM onset mode, the ECEI measurements alone cannot reveal to what extent these effects are related to the slowing down (and stopping) of the edge plasma rotation [30].

The duration of the temperature collapse phase differs for each ELM crash. Interestingly, the occurrence of filaments is only seen during this temperature collapse phase. With some of the first filaments seen moving in downward direction, it is uncertain to what extent their motion is related to the previously observed ELM onset mode or whether it could be determined by the plasma flows in the scrape-off-layer.

Compared to other observations, e.g. [11, 12] and [15], it is notable that the toroidal mode number $n$ of the off-mid-plane fluctuation presented here is much higher. However, these mode numbers have mostly been determined from a precursor that usually grows in amplitude in the last $\sim 100 \mu$ s and is seen in the Mirnov coils. This is obviously not the case here, since the off-mid-plane fluctuation does not develop into the actual crash. It is noted, though, that the $n$ number of the presented ELM onset mode is already lower than that of the off-mid-plane fluctuation. On the other hand, the existence of a 
high frequency $(300-500 \mathrm{kHz})$ precursor oscillation, which is often observed in the magnetics, is possibly connected to high- $n$ ballooning modes. It is also seen that these high frequency precursors drop in frequency during the final growth phase. Although most of the observed precursors are seen mainly in the magnetics, there are also examples that show the existence of a low frequency precursor that is not seen on the magnetics but only in the density [7].

Further study will be required to put these new observations in a broader perspective as well as in the context of the general ELM cycle as described in the introduction. In order to find the origin of the off-mid-plane fluctuation, a comparison will be made to the $T_{\mathrm{e}}$ oscillations observed in type-II ELMs, which show similar behaviour [31]. Combining the ECEI measurements of filaments during the temperature collapse phase with measurements from (visual ligth) cameras, Langmuir probes and e.g. the fast-ion-loss detector should resolve the different directions of motion observed. By slightly changing the magnetic field, or changing the settings of the diagnostic, the ECEI field of view will be shifted so the radial depth of the modes can be determined. It will also be investigated whether the observed phenomena occur in a similar way in other plasma conditions, e.g. different $q_{95}$ or edge collisionality. Finally, a direct comparison of the ECEI measurements of the mode observed at the ELM onset and the non-linear modelling of JOREK [10, 21, and 32] might also reveal whether or not this could indeed be the ELM trigger.

\section{Acknowledgement}

This work, supported by the European Communities under the Contract of Association between EURATOM-FOM, was carried out within the framework of the European Fusion Program, with financial support from NWO, FOM and EURATOM. The views and opinions expressed herein do not necessarily reflect those of the European Commission.

\section{References}

[1] Wagner F et al. 1982 Phys. Rev. Lett. 491408

[2] Zohm H 1996 Plasma Phys. Control. Fusion 38105

[3] Suttrop W 2000 Plasma Phys. Control. Fusion 42 A1

[4] Loarte A et al. 2003 J. Nucl. Mater. 313-316 962

[5] Suttrop W et al. 2007 'Active in-vessel coils and a conductive wall for MHD control in ASDEX Upgrade' Proc. $34^{\text {th }}$ EPS Conf. on Plasma Physics (Warsaw, Poland, 2007) vol 31F (ECA) P-5.119 (CD-ROM) http://epsppd.epfl.ch/Warsaw/pdf/P5_119.pdf

[6] Maggi C F 2010 Nucl. Fusion 50066001

[7] Oyama N et al. 2004 Nucl. Fusion 44582

[8] Snyder P B et al. 2002 Phys. Plasmas 92037

[9] Connor J W 1998 Plasma Phys. Control. Fusion 40191

[10] Huysmans G T A and Czarny O 2007 Nucl. Fusion 47659

[11] Bolzonella T et al. 2004 Plasma Phys. Control. Fusion 46 A143

[12] Terry J L et al. 2007 J. Nucl. Mater. 363-365 994

[13] Oyama N et al. 2011 Nucl. Fusion 51033009

[14] Kirk A et al. 2004 Phys. Rev. Lett. 92245002

[15] Maingi R et al. 2005 Nucl. Fusion 451066

[16] Koch B et al. 2007 J. Nucl. Mater. 363-365 1056

[17] Eich T et al. 2003 Phys. Rev. Lett. 91195003

[18] Eich T et al. 2005 Plasma Phys. Control. Fusion 47815

[19] Neuhauser J et al. 2008 Nucl. Fusion 48045005

[20] Jakubowski M W et al. 2009 J. Nucl. Mater. 390-391 781

[21] Pamela S et al. 2010 Plasma Phys. Control. Fusion 52075006

[22] Burckhart A et al. 2010 Plasma Phys. Control. Fusion 52105010

[23] Classen I G J et al. 2010 Rev. Sci. Instrum. 81 10D929

[24] Tobias B et al. 2009 Rev. Sci. Instrum. 80093502

[25] Park H K et al. 2004 Rev. Sci. Instrum. 753787

[26] Bornatici M 1982 Plasma Phys. 24629

[27] Fischer R et al. 2008 Fusion Sci. Technol. 58675 
[28] Schittenhelm M and Zohm H 1997 Nucl. Fusion 371255

[29] Kurzan B et al. 2007 Plasma Phys. Control. Fusion 49825

[30] Pütterich T et al. 2009 Phys. Rev. Lett. 102025001

[31] Wolfrum E et al. 2011 Plasma Phys. Control. Fusion 53085026

[32] Huysmans G T A et al. 2009 Plasma Phys. Control. Fusion 51124012 